\title{
Creating the Core Conditions of Extra-solar and Solar Giant Planets
}

P.M. Celliers ${ }^{1}$, J.H. Eggert',

G.W. Collins ${ }^{1}$, S. Brygoo ${ }^{1,2}$, R. Jeanloz ${ }^{3}$, R.S. McWilliams ${ }^{3}$, P. Loubeyre ${ }^{2}$, T.R. Boehly, J.E. Miller ${ }^{4}$

1

Lawrence Livermore National Laboratory 2

Département de Physique Théorique et Applications, CEA, Commissariat a ' I'Energie Atomique, 91680 Bruyères-le-Châtel, France 3

Department of Earth and Planetary Science, University of California, Berkeley, CA 94720

4 Laboratory for Laser Energetics, University of Rochester, Rochester, NY

\section{February 2007}




\section{Disclaimer}

This document was prepared as an account of work sponsored by an agency of the United States Government. Neither the United States Government nor the University of California nor any of their employees, makes any warranty, express or implied, or assumes any legal liability or responsibility for the accuracy, completeness, or usefulness of any information, apparatus, product, or process disclosed, or represents that its use would not infringe privately owned rights. Reference herein to any specific commercial product, process, or service by trade name, trademark, manufacturer, or otherwise, does not necessarily constitute or imply its endorsement, recommendation, or favoring by the United States Government or the University of California. The views and opinions of authors expressed herein do not necessarily state or reflect those of the United States Government or the University of California, and shall not be used for advertising or product endorsement purposes.

\section{Auspices Statement}

This work was performed under the auspices of the U. S. Department of Energy (DOE) by the University of California, Lawrence Livermore National Laboratory (LLNL) under Contract No. W-7405-Eng-48. The project (04-ERD-065) was funded by the Laboratory Directed Research and Development Program at LLNL. 


\title{
FY06 LDRD Final Report
}

\section{Creating the Core Conditions of Extra-solar and Solar Giant Planets}

\author{
LDRD Project Tracking Code: 04-ERD-065
}

\author{
Peter M. Celliers, Principal Investigator
}

\begin{abstract}
Materials can be experimentally characterized at high pressures and densities by sending a laser-induced shock wave through a sample that is pre-compressed inside a diamond-anvil cell. This combination of static- and dynamic-compression methods has been experimentally demonstrated, and ultimately provides access to the 10-100 TPa (0.1-1 Gbar) pressure range that is relevant to planetary science. We report on dynamical measurements of the high pressure compressibility of helium, hydrogen and helium/hydrogen mixtures up to $230 \mathrm{GPa}$ by combining laser shocks and static compression in diamond anvil cells. The initial density of samples in these precompressed targets has been varied by a factor of 3 . The measurements on the principal He Hugoniot, i.e with the initial density of cryo-helium, is extended above $100 \mathrm{GPa}$ and a maximum of compression ratio of greater than 5-fold of the initial density is observed. Also, a strong decrease in compressibility is observed by increasing the initial density. A similar data set has been produced for precompressed $\mathrm{H}_{2}$ and a mixture of $\mathrm{He}$ and $\mathrm{H}_{2}$.
\end{abstract}

\section{Introduction}

Peak pressures in the 1-10 TPa range exist inside large planets, with Earth's central pressure being $0.37 \mathrm{TPa}$ and "super-giant" planets expected to have central pressures in the 10-100 TPa range. The key to a credible planetary model is an accurate equation of state (EOS) for candidate mixtures of the elemental constituents of the planet, up to the extreme conditions of pressure and temperature achieved within planetary cores. However, experimental validation of EOS models at pressures more than a few Mbar $(0.1 \mathrm{TPa}=1 \mathrm{Mbar})$ has been obtained for only a few materials, and usually only along the principal Hugoniot, the locus of states accessible by a single shock wave[1]. In planets, brown dwarfs and lowest-mass stars, the interiors are fluid and convective, which means that interior thermodynamic states are distributed along an isentrope, except near thermal boundary layers (e.g., associated with major changes in composition).

Planetary isentropes reach much higher densities than can be achieved with standard shock-compression or static techniques. At planetary-core densities, material states are predicted to have quite unearthly properties, such as hightemperature superconductivity[2], and for planets more massive than $\sim 13$ Jupiter masses, thermonuclear fusion of deuterium[3]. A further complication relating to 
experimental methods for preparing material states relevant to planetary cores in the laboratory is that relevant mixtures $\left(\mathrm{He} / \mathrm{H}_{2}\right)$ cannot be formed at the low pressures (and cryogenic temperatures) typically used for target preparation for shock experiments.

To access the extreme densities of core conditions for relevant mixtures, we have developed diamond-anvil cell targets (see Fig. 1) for laser-shock compression experiments[4,5]. With this technique, the initial sample pressure is much higher than ambient (1-5 GPa), and the initial density may be from 1.5 to 10 times higher than ambient (depending on the sample): a huge increase compared to nonpressurized preparation methods. Furthermore, in the important case of $\mathrm{He} / \mathrm{H}_{2}$ mixtures pressurization is the only way to produce a uniform single-phase target sample for high pressure/density experiments. The higher initial density results in a significantly larger final density, and correspondingly lower temperature in the shock-compressed state.
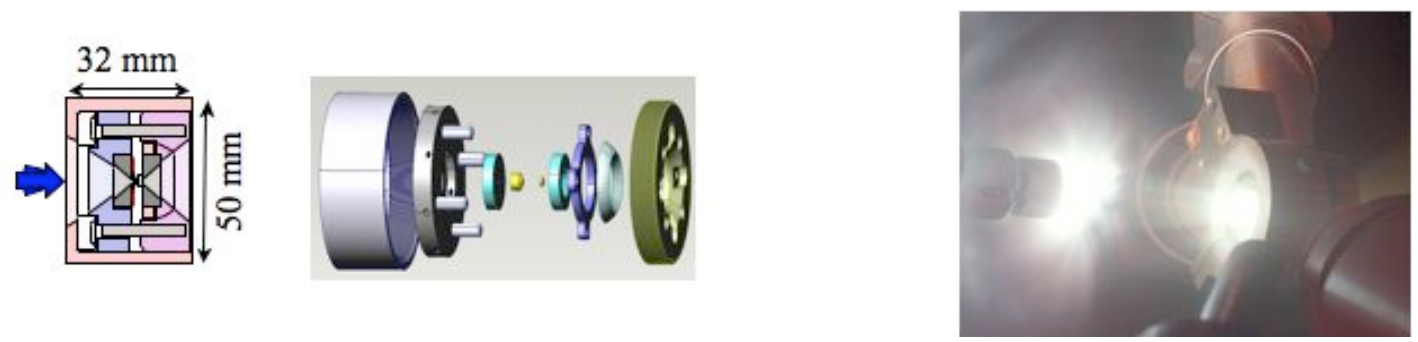

Figure 1. Schematic of diamond-anvil cell (left), showing both a cross-section (blue arrow indicates direction of incoming, shock-wave generating laser beams) and a pulled-apart view, and photograph (right) of a diamond cell as a laser-induced shock is being generated during an experiment at the Omega laser facility (University of Rochester).

Single- and multiple-shock Hugoniot techniques using pre-compressed targets can thus recreate deep-interior states of giant Solar-System planets, extra-solar planets, and even brown dwarfs and low-mass stars. Such equation-of-state measurements will provide crucial constraints on models that describe planetary structure (current internal state) and internal evolution, which is a key requirement for understanding the origins of planets. Electrical conductivity inferred from optical reflectance measurements can also be used as input data for models of planetary magnetic-field generation.

\section{Research Methods}

The dynamic-compression technique we have developed was initially demonstrated at the VULCAN laser facility at the Rutherford Appleton Laboratory in the UK $[4,5]$. Further refinements of the technique were developed by us as part of our research activities for this project, and these allowed us reliably to demonstrate it at OMEGA laser facility. Here we summarize the sample preparation, diagnostic measurement and data analysis methods we have developed during our efforts on this project.

\section{Sample preparation}

A sketch of the laser shock targets used in the present study is shown in Fig. 2. It is equipped with thin diamond windows, ranging from $100 \mu \mathrm{m}$ to $250 \mu \mathrm{m}$, on the drive laser side of the target and a sapphire anvil on the opposing side, facing the VISAR diagnostic. The drive side of the thin diamond was typically coated with a 
preheat shield ( $2 \mu \mathrm{m} \mathrm{Au})$ and a plastic ablator. On the sample chamber side, the thin diamond was flash Al coated and a quartz plate and a small ruby ball were placed on it. The target was loaded with the sample fluid $\left(\mathrm{He}, \mathrm{H}_{2}\right.$ or $\mathrm{He} / \mathrm{H}_{2}$ mixture) in a high pressure vessel. Determining the pressure-density-internal energy conditions in the initial state of the pre-compressed sample, $P_{0}, \rho_{0}$ and $E_{0}$ is essential to determine the $P, \rho$ and $E$ of the compressed state through the Rankine-Hugoniot equations and the measurement of the shock velocity $U_{s}$ and the particle velocity $u_{p}$ in shocked helium. Pressure was measured using the ruby fluorescence pressure scale[6]. The temperature shift of the reference was precisely corrected and an absolute precision of $\pm 0.03 \mathrm{GPa}$ could be achieved. $\rho_{0}$ and $\mathrm{E}_{0}$ were estimated by an accurate EOS of the sample[7]. The samples $\left(\mathrm{He}, \mathrm{H}_{2}\right.$ or $\left.\mathrm{He} / \mathrm{H}_{2}\right)$ were precompressed to pressures between $0.12 \mathrm{GPa}$ and $12.5 \mathrm{GPa}$ (which span the range of densities ranging from liquid the cryogenic density $\rho_{L}\left(0.1235 \mathrm{~g} / \mathrm{cm}^{3}\right.$ for $\mathrm{He}, 0.075$ $\mathrm{g} / \mathrm{cm}^{3}$ for $\mathrm{H}_{2}$ ) to more than $3 \rho_{\mathrm{L}}$. The thickness of He in between the quartz and the anvil, i.e the transit gap for the shock wave propagating in $\mathrm{He}$, and the thickness of quartz were accurately determined by interferometric measurements and accurate refractive index determinations for $\mathrm{He}$ and $\mathrm{H}_{2}[8]$. The initial pressure effect on the density and refractive index of quartz were also taken into account $[9,10]$.

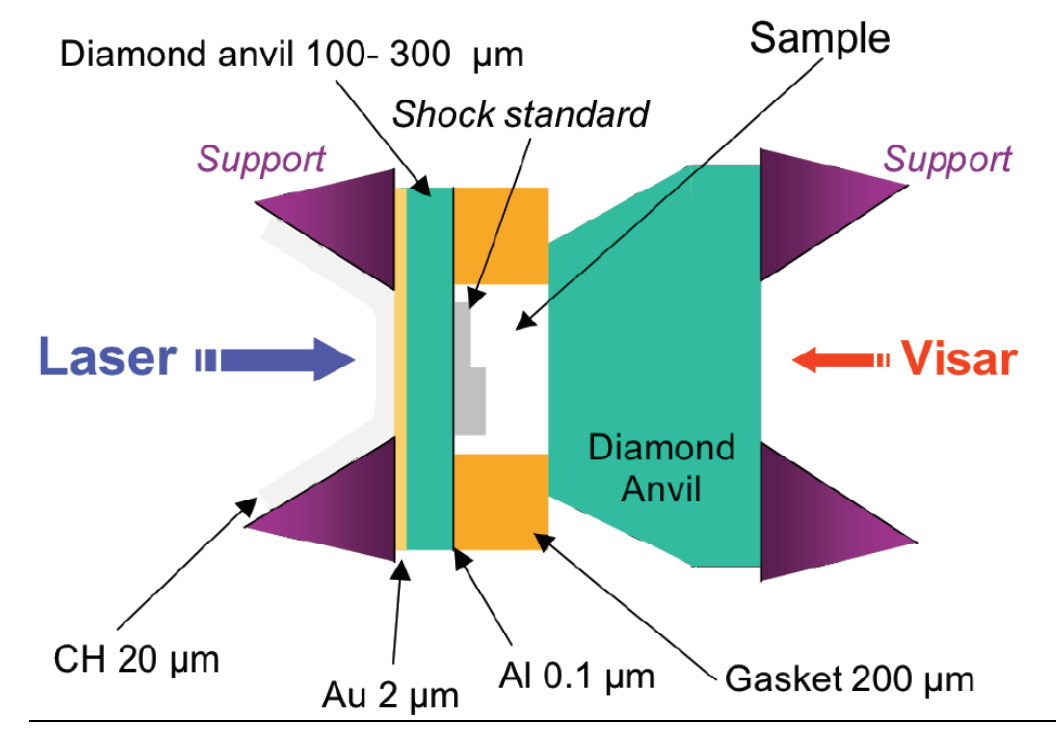

Figure 2. Schematic cross-section of diamond anvils and sample, with the drivelaser that creates the shock wave entering from the left. Supports for the anvils are shown in purple and, as described in the text, current laser systems require the anvil on the shock-entry side to be thin. The sample is indicated, along with a shock-wave standard (an Al plate in this sketch). Diagnostics described in the text (VISAR and not shown - pyrometry) record the dynamic compression of the sample through the second anvil.

\section{Laser drive conditions}

Up to 6 beams were used to generate the shock in a 1 ns pulse, with an energy up to $1 \mathrm{~kJ}$ focused to a $650 \mu \mathrm{m}$ circular spot and so with a maximum flux onto the target of $6 \times 10^{14} \mathrm{~W} / \mathrm{cm}^{2}$. At the OMEGA facility the 6 beams strike the sample at approximately 20 degrees angle of incidence. All other beams at the OMEGA facility enclose larger angles relative to the target surface normal, and consequently cannot be used owing to the confined geometry of the cell. As a consequence of the limited laser drive energy available with the OMEGA facility, the samples could be pre-compressed up to no more than $1-5 \mathrm{GPa}$, because the 
diamond anvil from which the shock-front enters needs to be thin, no more than about 100-400 $\mu \mathrm{m}$ thick. This amounts to little more than a microscope-slide cover slip, albeit made of diamond. The limitation of the available set of beams, the twodimensional nature of shock generation within the confined geometry of the cell, and impedance matching from the stiff diamond window into the compressible sample all limit the peak shock pressures achievable in the sample to less than $\sim 250 \mathrm{GPa}$. Nevertheless, in the case of He this peak exceeds the single shock peak pressure previously achieved on He samples by a factor greater than 10 .

\section{Diagnostics}

Measurement of the wave velocities in the sample is accomplished by velocity interferometry, a line-imaging VISAR diagnostic $[11,12]$, that provides a record of the shock and material (particle) velocities inside the sample chamber (Fig. 3). Briefly, VISAR operates by illuminating the sample with a single-frequency laser and imaging the reflected light through an interferometer onto a detector. The interferometer is configured to have unequal paths: a change in the frequency of the light passing through it causes a change in the fringe phase in proportion to the Doppler shift in frequency. The velocity of moving reflectors in the target (interfaces and shock fronts) are thereby measured to $\sim 1 \%$ precision. If the initial thickness of the (precompressed) sample is known, a measurement of the shock-wave transit time determines the shock velocity.

In addition to the Doppler shift measurement, the VISAR diagnostic affords the measurement of two other observables. Firstly, the optical reflectivity of the reflecting shock front can be determined from the intensity of the light reflected from the shock front. Secondly, the optical train of the VISAR diagnostic allows the collection of the thermal emission radiated from the shock front, which is typically reaches temperatures of 0.5 to $6 \mathrm{eV}$ in our experiments. This emission occupies the visible wavelength band, and is recorded on an optical streak camera. The emission intensity can then be related to an effective temperature or brightness temperature of the shock compressed sample. The brightness temperature can be corrected for the sample emissivity to yield the sample temperature because the reflectivity measurement provides information on the emissivity. 

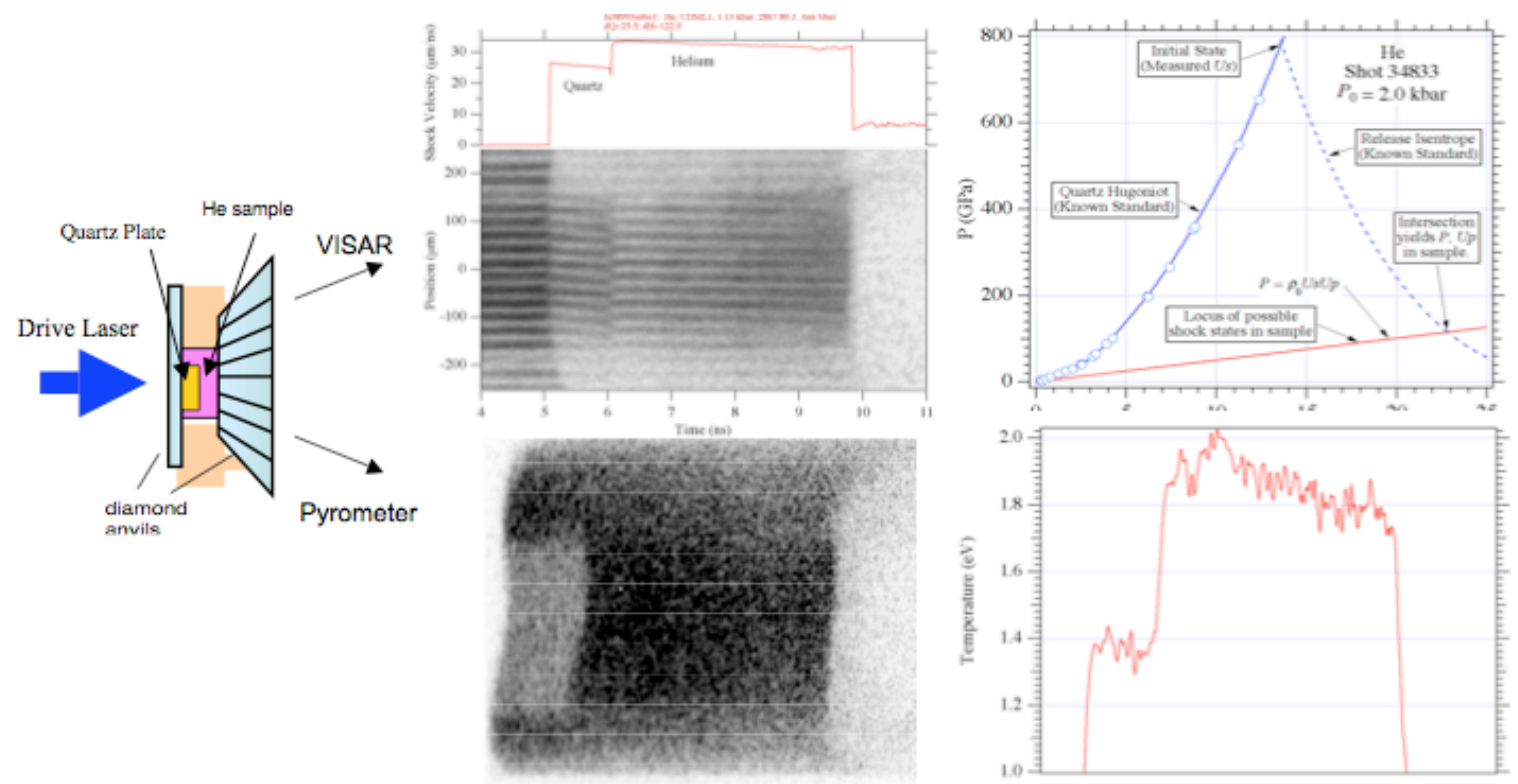

Figure 3. Example of the data records obtained in a typical experiments. The VISAR recording (upper left image) produces the fringe patterns which are analyzed to produce a continuous measurement of shock velocities in the quartz plate and in the sample. These velocities are then used as parameters for impedance match calculations to determine the equation of state (upper right plot). The pyrometer record (lower left image), obtained simultaneously, yields a measurment of the luminous emission from the shock front in the target, first as it passes through the quartz, later as it passes through the sample. The temperature in the sample is determined relative to that in the quartz, which has been previously calibrated.

A key component of this diagnostic method is the quartz plate which was used for the impedance matching analysis (described below) as well as for the relative determination of the temperature and reflectivity. The sample reflectivity, $R_{\lambda}$, was determined relative to the reflectivity of the shock front in the quartz plate. Similarly the emission from the sample relative to that in the quartz plate, from which a brightness temperature was extracted. The brightness temperature was corrected for the shock front emissivity, by assuming a wavelength indepedndent emissivity $\varepsilon=1$ $\mathrm{R}_{\lambda}$ to arrive at a sample temperature. These relative determinations are prefereable and more accurate than absolute measurements because the constricted optical geometry of the sample cell make it very difficult to obtain accurate absolute calibrations for these measurements. On the other hand absolute measurements of the brightness temperature and reflectivity on shock fronts in quartz have been performed previously in a separate set of measurements, thus enabling our use of the quartz plate as a reference standard for not only for the impedance matching, but also for reflectivity and brightness temperature measurements.

\section{Impedance matching for EOS determination}

The $P, \rho$ of the shocked sample are determined from the velocity of the shock front, $U_{s H e}$ and the He particle velocity, $u_{p H e}$ using Rankine-Hugoniot relations, $P=\rho_{0} \quad U_{s} U_{p}$ and $\rho=\rho_{0} U_{s} /\left(U_{s}-U_{p}\right)$, where $\rho_{0}$ is the initial density. To determine $\mathrm{u}_{\mathrm{pHe}}$ from $\mathrm{U}_{\mathrm{sQ}}$ and $\mathrm{U}_{\mathrm{sHe}}$ we use standard impedance matching techniques, which are discussed in detail in Ref.[13], and summarized briefly in the following. In our experiments we use quartz as the reference standard. The impedance matching construction requires the knowledge of the quartz Hugoniot and its off-Hugoniot 
states along release isentropes. The Hugoniot of quartz is now very well determined[14]. A well-known practical approximation for the off-Hugoniot is a graphical construction which approximates the release profile by the mirror reflection of the principal Hugoniot in the pressure vs particle velocity plane. The correction to this approximation can then be estimated by using a detailed equation of state model for quartz. For all of our experiments the shocked quartz was strong enough to transform it into a conducting dissociated fluid[15]. We have used a Mie-Gruneisen model with $\Gamma=0.66$, valid in this dense fluid domain, to generate a correction to the reflected shock were made with it. Similar corrections were obtained by using the Sesame tables and a recent model of silica[16]. The Gruneisen model has the advantage that there is no interpolation errors that can occur with tabulated EOS and it allows to propagate measurement uncertainties. Finally, the effect of precompression changes slightly the density of quartz and consequently the Hugoniot of quartz. But that can be taken into account since the Hugoniot of various forms of silica have been observed to be parallel in the dense fluid state, with a shift depending only on the initial density[17].

\section{Results/Technical Outcome}

During the course of this project we performed approximately 30 experiments on diamond anvil cells filled with $\mathrm{H}_{2}, \mathrm{He}$ and $\mathrm{He} / \mathrm{H}_{2}$ mixtures. All experiments were carried out at the OMEGA laser facility. As describe above, most of these experiments produced quantitative data on the Hugoniot parameters $(P, V$ and $E)$, temperature $(T)$ and shock front reflectivity (which can be related to electrical conductivity, $\sigma)$.

\section{Results on He}

The results produced by these experiments have significantly extended the boundaries of our knowledge of the phase diagram of dense He. This data set provides measurements of the properties of dense He to the highest pressures yet, greater than $200 \mathrm{GPa}$. In particular we have compressed $\mathrm{He}$ more than 13-fold relative to the the cryogenic liquid density, and have obtained conducting states in dense He plasmas. The insulator-conductor transition in He has been found to be two orders lower in pressure than that determined by low temperature band structure calculations.

The Hugoniot data shows evidence for a softening of the Hugoniot at particle velocities $u_{p}>10 \mathrm{~km} / \mathrm{s}$. This softening is consistent with the onset of thermallyactivated ionization. The peak compression reaches greater than 5-fold for initial densities equal to the cryogenic liquid density. Lower peak compressions result for high initial densities. Both the compression data and the temperature data are consistent with existing equation of state models for dense He. The reflectivity data show a strong correlation with the temperature indicating that the ionization which causes the rise in reflectivity a thermally-activated process.

We have fit the reflectivity data to a simple semiconductor model, which assumes a density dependent activation (or gap) energy. From the fit we estimate the gap energy to be approximately $15 \mathrm{eV}$ at densities near $1 \mathrm{~g} / \mathrm{cm}^{3}$. From this model we estimate that the electrical conductivity of our pre-compressed and shocked samples reached as high as $500(\Omega-\mathrm{cm})^{-1}$. This conductivity is typical of a semiconductor; extrapolation of this model to the Jupiter isentrope suggests that metallic He conditions will be obtained near 300 GPa along the Jupiter isentrope. Laboratory re-creation of these conditions may be attained if we can compress a $7 x$ $\rho\llcorner$ sample to a pressure of 300 GPa (currently beyond the capabilities of the OMEGA laser, but within the capabilities of NIF). 

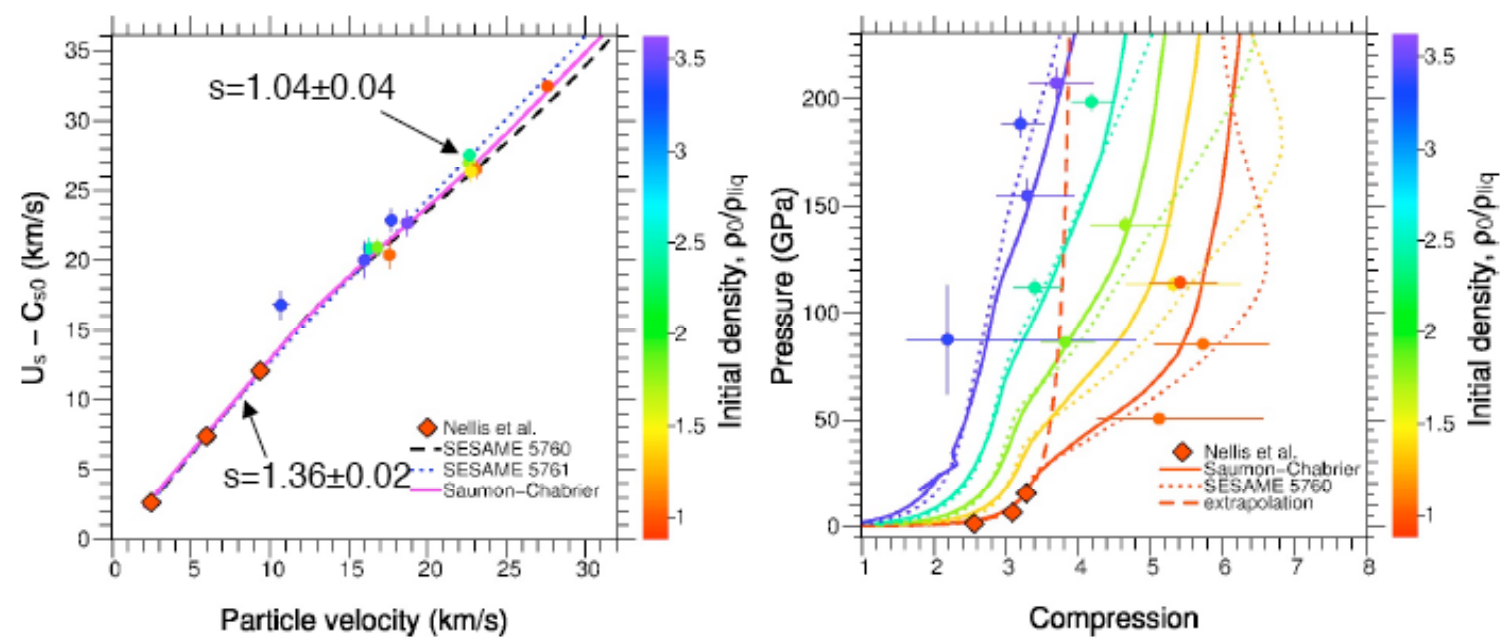

Figure 4. Shock Hugoniot data for pre-compressed He samples. The left frame shows the standard $U_{s}$ versus $u_{p}$ plot of the Hugoniot, except that we plot $U_{s}-C_{s 0}$ where $c_{s 0}$ is the sound speed of the initial state. It can be shown that in this representation the data fall on a common curve. The right frame shows the Hugoniot data in the pressure-compression plane. In both frames the initial density relative to cryogenic liquid density is indicated by the colors of the curves (various models) and the data points, with the mapping indicated by the color scale on the right of each frame. The early gas-gun data is indicated by the diamonds.

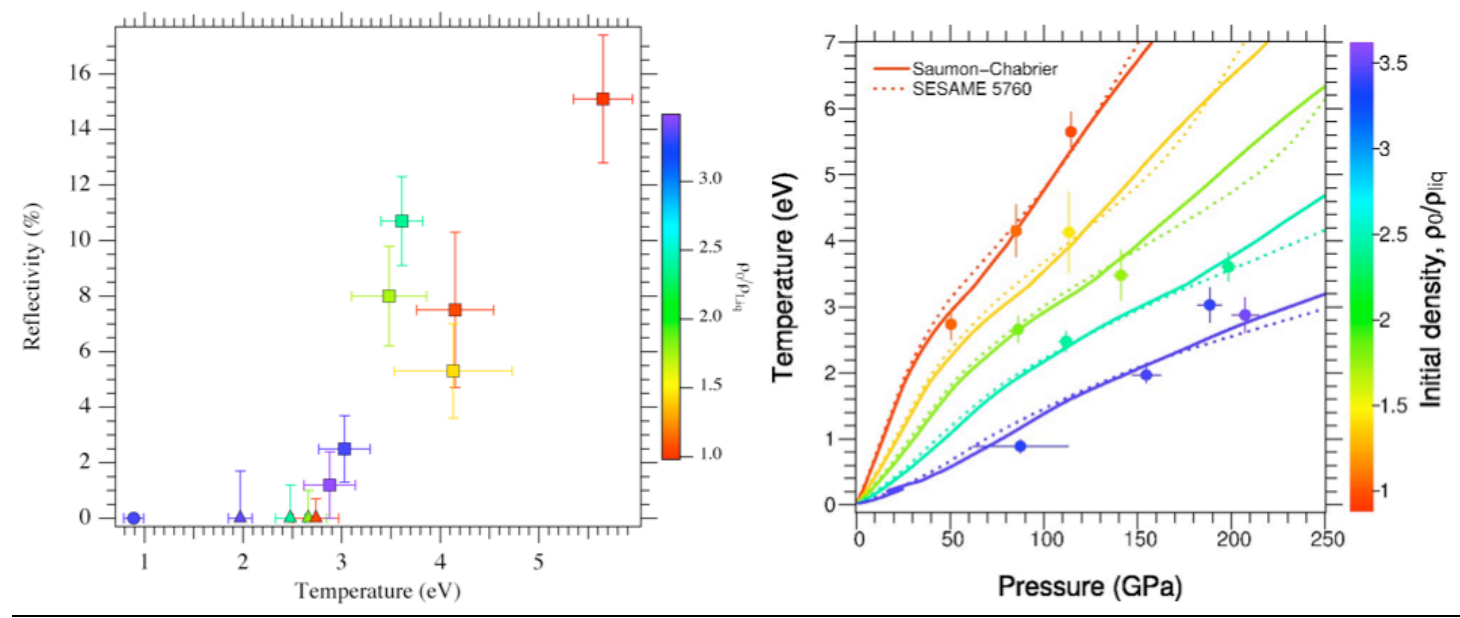

Figure 5. Measured dependence of shock front reflectivity (left frame) and shock front temperature (right frame) for the experiments on He. 


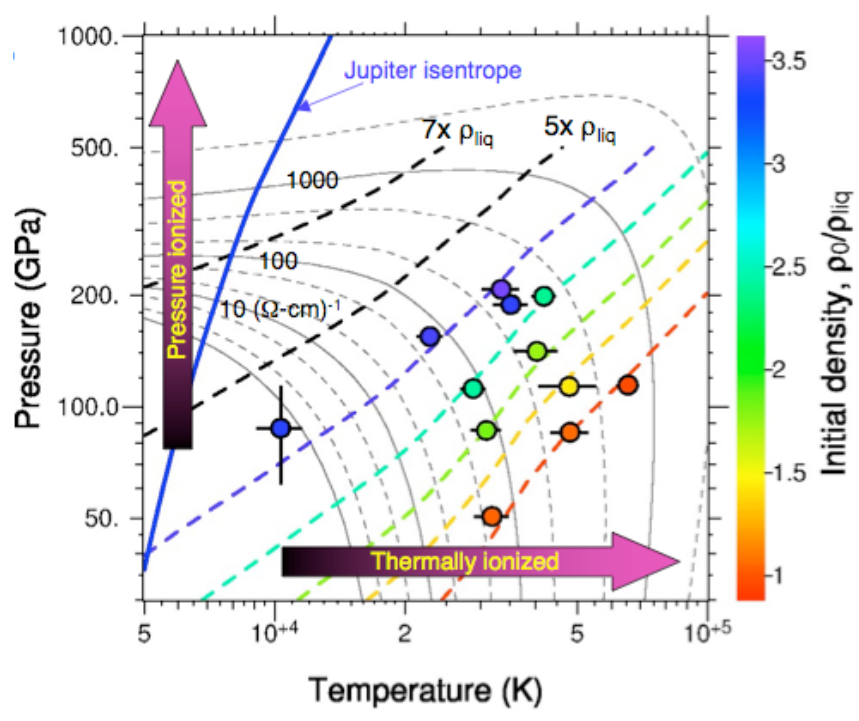

Figure 6. Electrical conductivity of dense $\mathrm{He}$ on the pressure-temperature plane (gray solid and dashed contours with labels $\left.10,100, \& 1000(\Omega-\mathrm{cm})^{-1}\right)$ as estimated from a semiconductor model fit to the reflectivity data. The circles show the data points on the P-T plane for which the model is well-constrained. The thick dashed curves show the loci of several pre-compressed hugoniot curves including those accessed in our experiments (colored curves) and in future experiments (black). The Jupiter isentrope is also indicated.

\section{Results on $\mathrm{H}_{2}$}

We also collected a significant data set on $\mathrm{H}_{2}$ at different initial densities. These new data provide an interesting comparison to the current extensive data set on cryogenic liquid deuterium. Initial analysis of these data suggest that the $\mathrm{H}_{2}$ hugoniot may be more compressible than the $D_{2}$ hugoniot. Further analysis, which incorporate the results of more recent experiments is ongoing.

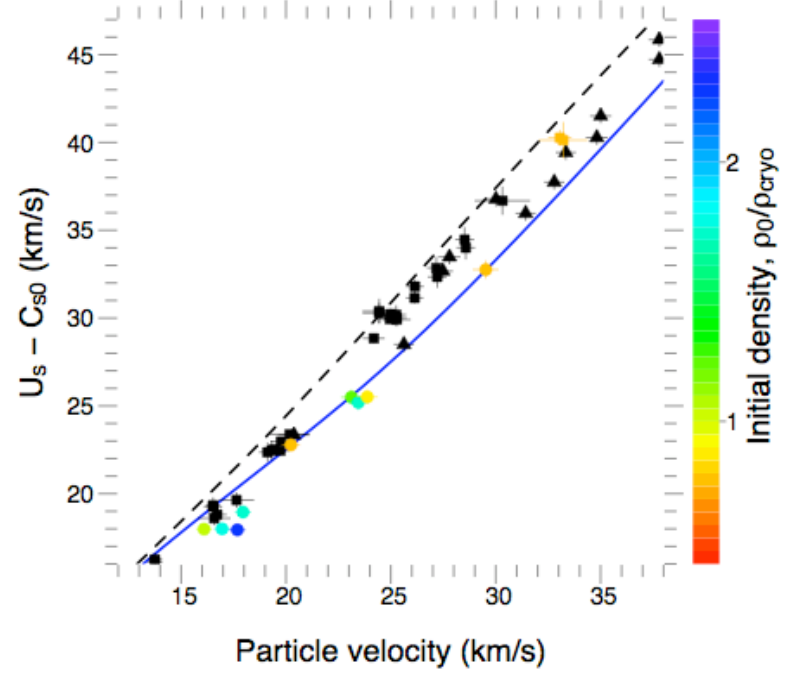

Figure 7. Shock Hugoniot data for pre-compressed $\mathrm{H}_{2}$ samples, shown on the Us-up plane, with the initial sound speed subtracted from the Us, similar to the representation in Fig. 4 for He. The black symbols show Hugoniot data for cryogenic $D_{2}$ collected at OMEGA and $Z$; these latter data have been scaled to map to the $\mathrm{H}_{2}$ hugoniot. 


\section{Results on $\mathrm{He} / \mathrm{H}_{2}$ mixture}

Finally, we have produced, for the first time, several data points on a $50 \%$ $\mathrm{He} / \mathrm{H}_{2}$ mixture. The preliminary Hugoniot results indicate that the compressibility of the mixture is consistent with an idealized mixing model based on EOS's of pure $\mathrm{He}$ and $\mathrm{H}_{2}$.

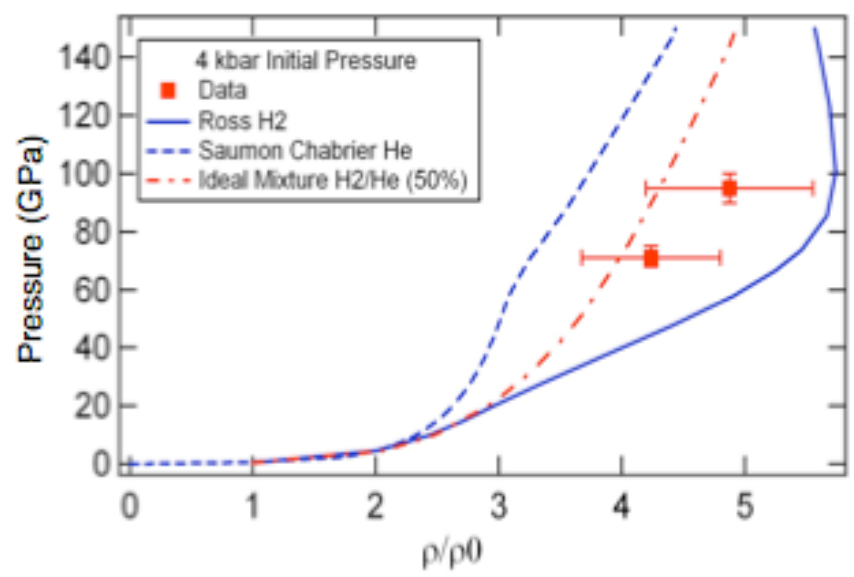

Figure 8. Shock Hugoniot data for pre-compressed $\mathrm{He} / \mathrm{H}_{2}$ mixture samples. Preliminary analysis indicates that the compressibility of the mixture lies intermediate between the compressibilities of the pure phases.

\section{Exit Plan}

In the near term the techniques developed in this project are seeing continued application in an ongoing National Laser User Facility (NLUF) project at the OMGEA laser. This project is expected to continue through the end of FY08, encompassing another 15 - 20 experiments. In the longer term we expect the techniques developed in this project will have a bright future, and will lead to continued improvements in our methods, as well as further exploration on future high energy laser facilities. In particular a dedicated series of shots on the CEA LIL facility is planned in 2008, and plans for fielding this technique on NIF under the NIF University Use program are being developed with the aim of fielding the first experiments in 2009.

\section{Summary}

We have demonstrated a new technique for characterizing the properties of dense low- $Z$ fluids relevant to the understanding of the planetary cores. The successful application of this technique at the OMEGA laser facility has produced a wealth of new data on the properties of dense $\mathrm{He}, \mathrm{H}_{2}$ and $\mathrm{He} / \mathrm{H}_{2}$ mixtures. Future application of this technique on larger facilities such as NIF should extend our knowledge of these materials well into the TPa pressure range.

\section{Acknowledgements}

We acknowledge the help of the Chuck Sorce (of LLNL stationed at LLE), Jack Armstrong and Greg Pien as well as the operations crew of the OMEGA laser facility. In addition we acknowledge the help of Walt Unites of LLNL in preparing the quartz reference samples, and Jim Cox of LLNL for designing and fabricating the target manipulator. 


\section{References}

1. Eremets, M. I. (1996) High Pressure Experimental Methods (Oxford Univ. Press, Oxford, UK).

2. Ashcroft, N.W., Phys. World 8, 43 (July, 1995).

3. Ishimaru, S., Rev. Mod. Phys. 65, 255 (1993).

4. Loubeyre, P., Celliers, P.M., Hicks, D. G., Henry, E., Dawaele, A., Pasley, J., Eggert, J., Koenig, M., Occelli, F., Lee, K.K. M., Jeanloz, R., Neely, D., BenuzziMounaix, A., Bradley, D., Bastea, M., Moon, S. \& Collins, G. W. High-Pressure Research, 24, 25-31 (2004).

5. Lee, K. K. M., Benedetti, L. R., Jeanloz, R., Celliers, P. M., Eggert, J. H., Hicks, D. G., Moon, S. J., Mackinnon, A., Da Silva, L. B., Bradley, D. K., Unites, W., Collins, G. W., Henry, E., Koenig, M., Benuzzi-Mounaix, A., Paley, J. \& Neely, D. J. Chem. Phys. 125, 014701 (2006).

6. Mao, H.K., Xu, J., and Bell, P.M. J. Geophys. Res. B 91, 4673 (1986).

7. LeToullec, R. Loubeyre, P. and Pinceaux, J.P. Phys. Rev. B 40, $2368(1989)$.

8. Dewaele, A., Eggert, J., Loubeyre, P. and LeToullec, R. Phys. Rev. B 67, 094112 (2003).

9. Meister, E., Robertson, E.C., Were, R.W. and Raspet, R. J. Geophys. Res. 85, 6461 (1980).

10. Vedman, K. and Davis, J.A. J.O.S.A. 57, 1140 (1967).

11. Barker, L. M. \& Hollenbach, R. E. J. Appl. Phys., 43, 4669-4675 (1972).

12. Celliers, P. M., Bradley, D. K., Collins, G. W., Hicks, D. G., Boehly, T. R. \& Armstrong, W. J., Rev. Sci. Instrum. 75, 4916-4929 (2004).

13. Celliers, P.M., ...

14. Hicks, D.G., Boehly, T.R., Celliers, P.M., Eggert, J.H., Vianello, E., Meyerhofer, D.D., and Collins, G.W. Phys. Plasmas, 12, 082702 (2005).

15. Hicks, D.G., Boehly, T.R., Eggert, J.H., Miller, J.E., Celliers, P.M. and Collins, G.W. Phys. Rev. Lett. 97, 025502 (2006).

16. G. Kerley. Sandia Technical report (2001).

17. R.F. Trunin. Sov. Phys. Upsekhi 37, 1123 (1994). 